\title{
No-Hair Theorem for Black Holes in Astrophysical Environments
}

\author{
Norman Gürlebeck* \\ Center of Applied Space Technology and Microgravity (ZARM), \\ University of Bremen, Am Fallturm, 28359 Bremen, Germany, EU
}

(Received 27 August 2014; revised manuscript received 13 November 2014; published 15 April 2015)

\begin{abstract}
According to the no-hair theorem, static black holes are described by a Schwarzschild spacetime provided there are no other sources of the gravitational field. This requirement, however, is in astrophysical realistic scenarios often violated, e.g., if the black hole is part of a binary system or if it is surrounded by an accretion disk. In these cases, the black hole is distorted due to tidal forces. Nonetheless, the subsequent formulation of the no-hair theorem holds: The contribution of the distorted black hole to the multipole moments that describe the gravitational field close to infinity and, thus, all sources is that of a Schwarzschild black hole. It still has no hair. This implies that there is no multipole moment induced in the black hole and that its second Love numbers, which measure some aspects of the distortion, vanish as was already shown in approximations to general relativity. But here we prove this property for astrophysical relevant black holes in full general relativity.
\end{abstract}

DOI: 10.1103/PhysRevLett.114.151102

PACS numbers: 04.20.Cv, 04.20.Ha, 04.20.Jb, 04.70.Bw

Introduction.-The no-hair theorem states that any isolated static black hole is necessarily a Schwarzschild black hole and that there is only one free parameter describing the spacetime- the mass $M$. Although the black hole has actually one hair, $M$, this property is still called the no-hair theorem and, thus, the black hole is called bald. This theorem is, of course, very appealing for astrophysics, since just one parameter has to be measured to determine the entire spacetime around a black hole. Even if the black hole rotates, a similar theorem holds and only the mass and the spin have to be measured.

In fact, these two parameters are already observable, see, e.g., Refs. [1-3]. Additionally, new observatories like GRAVITY (see Ref. [4]) will improve these measurements further. An independent and promising approach is the measurement of the shadow of a black hole, see, e.g., Refs. [5,6] for recent results. The shadow will be resolvable in the millimeter and submillimeter range with the Event Horizon Telescope [7]. Moreover, the potential discovery of a binary system containing a black hole promises headway for characterizing black holes either via pulsar timing if the companion is a pulsar or via the detection of gravitational waves.

Since the no-hair theorem dictates in the rotating case that the quadrupole moment of a black hole is determined by its mass and spin, an independent measurement of all three parameters allows for a test of alternative theories of gravity or of the assumptions of the no-hair theorem, for recent approaches see, e.g., Refs. [8-10]. For example, the source could be described by matter distributions like boson stars for which the no-hair theorem does not hold.

A crucial assumption for the no-hair theorem is that the black hole is isolated; i.e., the spacetime is asymptotically flat and contains no other sources. However, in many astrophysical situations this requirement is not fulfilled, e.g., for black holes in binary systems, if the black hole is surrounded by plasma, an accretion disk, or if jets are formed in its vicinity; i.e., it might put on different types of wigs. These additional sources contribute also to the total multipole moments of the spacetime. Hence, a formulation like the standard no-hair theorem for isolated black holes cannot hold anymore, whereas a formulation solely for the part of the total multipole moment sourced by the black hole might still be correct. The latter is shown in this Letter. In the exterior field of additional sources, the black hole is distorted and the inner geometry of the horizon changes. This is measured by the Love numbers of the first kind or the multipole moments of isolated horizons, see Refs. [11-13]. Nonetheless, we show here that this does not imply that the black holes are not anymore bald. More precisely: Although the total multipole moments of the spacetime measured at infinity change, this is solely due to the external sources and not to a different contribution of the black holes themselves. In fact, distorted black holes have only a mass monopole. Thus, even though the black hole might put on a wig it still looks bald. Note that we assume here for simplicity static black holes.

Distortions of static black holes and neutron stars are of particular interest for inspirals treated in an adiabatic regime, see, for details on the validity of this regime, Refs. $[14,15]$. In such a quasistatic approximation, the black hole or the neutron star is distorted due to the external field of the companion instantaneously. Thus, the system is additionally axially symmetric with respect to the axis joining the two constituents of the binary system and the metric of distorted black holes of Ref. [16] is applicable. The imprints of such distortions in the gravitational waves emitted by inspiraling binaries give information on the 
equation of state of neutron stars [14,17]. Moreover, those imprints can be used to experimentally reveal if a constituent of a binary system is a black hole. This provides an avenue along which the existence of black holes can be directly inferred. On the other hand, if the existence of a black hole in a binary system is established independently by observing, say, gamma ray bursts at later stages of the inspiral, then the measurement of its distortions using gravitational waves allows us to test general relativity via the here presented no-hair theorem.

The distortions of the black holes and neutron stars are characterized by the Love numbers of first and second kind, $h_{r}$ and $k_{r}$, cf. Refs. $[15,18,19]$. Roughly speaking, the $h_{r}$ measure the changes in the shape of the horizon or the neutron star and the $k_{r}$ measure the change in the asymptotic multipole moments caused by the distortion due to an external source, see Refs. [13,15,19-21] for their use in general relativity. In the latter four works, it was established using approximation methods that the $k_{r}$ vanish for fourdimensional black holes. However, it was debated if this result is still valid in the case higher orders in the approximations scheme are taken into account. We resolve this dispute here by proving the result analytically without any approximation. Although these distortions are not crucial to detect gravitational waves in prospective data from Advanced LIGO and Advanced Virgo [22], they will be important for a detailed analysis of the data and for future detectors with an increased sensitivity, see, e.g., Refs. [23,24].

The Love numbers of the second kind were also applied to establish universal relations, i.e., relations that are independent of the equation of state, between certain physical parameters describing neutron stars, see Refs. [25-27] but also Refs. [28-31]. The here considered black hole case is solved analytically in full general relativity and, thus, it serves as a test for the various approximation schemes employed for neutron stars also in this respect.

Subsequently, we use geometric units, in which $G=c=1$, where $c$ is the velocity of light and $G$ Newton's gravitational constant. The metric has the signature $(-1,1,1,1)$. Greek indices run from 0 to 3 and Latin indices run from 1 to 3 .

Distorted black holes.-The metric of arbitrary static and axially symmetric spacetimes can be written in the Weyl form under standard assumptions, cf. Ref. [32]:

$d s^{2}=e^{2 k-2 U}\left(d \rho^{2}+d \zeta^{2}\right)+W^{2} e^{-2 U} d \varphi^{2}-e^{2 U} d t^{2}$,

where the functions $U, k$, and $W$ depend on $\rho$ and $\zeta$. Note that the metric functions $U$ and $W$ can be expressed by the timelike Killing vector $\xi^{\alpha}$ and the spacelike Killing vector $\eta^{\alpha}$ :

$$
e^{2 U}=-\xi_{\alpha} \xi^{\alpha}, \quad W^{2}=-\eta_{\alpha} \eta^{\alpha} \xi_{\beta} \xi^{\beta} .
$$

In the case that the exterior sources are static and axially symmetric or allow for a quasistatic description, the general metric near the horizon $\mathcal{H}$ of a distorted black hole was found by Geroch and Hartle in Ref. [16] in the form of Eq. (1). In a neighborhood of $\mathcal{H}$, we assume pure vacuum, which is physically reasonable if the matter can be treated quasistatically and satisfies the energy conditions, cf. Ref. [33]. Thus, there exists a surface $\mathcal{S}_{\mathcal{H}}$, which encloses $\mathcal{H}$ and no other sources. If $\mathcal{S}_{\mathcal{H}}$ is sufficiently close to $\mathcal{H}$, the metric functions in Eq. (1) read between $\mathcal{S}_{\mathcal{H}}$ and $\mathcal{H}$

$$
U=U_{S}+U_{D}, \quad k=k_{S}+k_{S D}, \quad W=\rho .
$$

The functions $U_{S}$ and $k_{S}$ are given by the respective quantities of the Schwarzschild black hole:

$$
\begin{aligned}
U_{S} & =\frac{1}{2} \log \left[\frac{r_{+}+r_{-}-2 M}{r_{+}+r_{-}+2 M}\right], \\
k_{S} & =\frac{1}{2} \log \left[\frac{\left(r_{+}+r_{-}\right)^{2}-4 M^{2}}{4 r_{+} r_{-}}\right], \\
r_{ \pm}^{2} & =\rho^{2}+(\zeta \pm M)^{2} .
\end{aligned}
$$

Subsequently, we find that the parameter $M$ coincides with the Komar mass of the distorted black hole. The function $U_{D}$ is determined by the exterior matter and it solves a Laplace equation,

$$
\left(\frac{\partial^{2}}{\partial \rho^{2}}+\frac{1}{\rho} \frac{\partial}{\partial \rho}+\frac{\partial^{2}}{\partial \zeta^{2}}\right) U_{D}=0
$$

If $U_{D}$ vanishes, the spacetime describes a Schwarzschild black hole. The function $k_{S D}$ follows from a line integration once $U_{D}+U_{S}$ is known, cf. Ref. [32]. However, we do not require its explicit form subsequently.

The horizon of the distorted black hole is located at the symmetry axis $(\rho=0, \zeta \in[-M, M])$ like for the Schwarzschild black hole. In fact, in canonical Weyl coordinates the horizon can always be located at $\rho=0$, see Ref. [34]. These coordinates allow a shift in the $\zeta$ coordinate. We employed this freedom to place the horizon symmetrically with respect to that coordinate, i.e., that the "north and south pole" of the horizon are characterized by $\zeta_{N / S}= \pm M$, respectively. At these points, $U_{D}$ has to take the same value to avoid struts, which we want to exclude for simplicity, see Ref. [16]. If the external matter is reflection symmetric, like for accretion disks or jets, this is trivially satisfied. Note that the metric functions take the form of Eqs. (3)-(5) only in a neighborhood of $\mathcal{H}$ and they neither describe directly the asymptotic behavior nor the metric in the interior of the external source. Nonetheless, we will be able to conclude with the help of the source integrals the contributions of the distorted black hole to the asymptotic multipole moments without specifying the exterior sources in detail. These could also include other black holes. We only require that the spacetime is asymptotically flat and that all external sources are contained in a region, which 
does not contain $\mathcal{H}$ and which does not extend to infinity. We denote its boundary by $\mathcal{S}_{\text {ext }}$.

The source integrals.-To disentangle the contributions of the black hole and the external sources to the asymptotic multipole moments, the source integrals proved to be the essential tool. They were recently derived in Ref. [35] and they make it possible to define the asymptotics of the spacetime including the Geroch multipole moments by evaluating quasilocal surface or volume integrals. The respective surfaces and volumes need only to envelop or contain all regions with a nonvanishing stress-energy tensor. Here we need the surface integrals and introduce the required quantities, subsequently.

The Weyl multipole moments $U^{(r)}$ are defined as the expansion of $U$ along the axis of symmetry close to infinity, i.e.,

$$
U=\sum_{r=0}^{\infty} \frac{U^{(r)}}{|\zeta|^{r+1}}
$$

As we will see later the coordinate $\zeta$ can be defined geometrically so that this definition is also covariant. Indeed, it was shown in Ref. [36] that from the $U^{(r)}$ the Geroch multipole moments $m_{r}$ can be determined uniquely by nonlinear algebraic relations. To calculate the $m_{r}$, the $U^{(k)}$ need to be known for $0 \leq k \leq r$. Thus, it is sufficient for us to consider here the $U^{(r)}$. Note that the origin with respect to which the multipole moments are measured was chosen by requiring $\zeta_{N / S}= \pm M$.

Furthermore, we use the functions

$$
\begin{aligned}
& N_{-}^{(r)}(x, y)=\sum_{k=0}^{\left\lfloor\frac{r}{2}\right\rfloor} \frac{2(-1)^{k+1} r ! x^{2 k+1} y^{r-2 k}}{4^{k}(k !)^{2}(r-2 k) !}, \\
& N_{+}^{(r)}(x, y)=\sum_{k=0}^{\left\lfloor\frac{r-1}{2}\right\rfloor} \frac{2(-1)^{k+1} r ! x^{2 k+2} y^{r-2 k-1}}{4^{k}(k !)^{2}(r-2 k-1) !(2 k+2)} .
\end{aligned}
$$

It can easily be checked that these functions obey the equations

$$
N_{+, x}^{(r)}-N_{-, y}^{(r)}=0, \quad N_{+, y}^{(r)}+N_{-, x}^{(r)}-\frac{N_{-}^{(r)}}{x}=0 .
$$

Commas denote partial derivatives. Additionally, let us introduce the 1-form

$$
Z_{\alpha}=\epsilon_{\alpha \beta \gamma \delta} W^{, \beta} W^{-1} \eta^{\gamma} \xi^{\delta},
$$

where $\epsilon_{\alpha \beta \gamma \delta}$ is the volume form of the spacetime. In vacuum, $Z_{\alpha}$ is exact and it is hypersurface orthogonal in the entire spacetime. Since the surfaces of interest, $\mathcal{S}_{\mathcal{H}}$ and $\mathcal{S}_{\text {ext }}$, lie in the vacuum region or its boundaries, we can introduce a scalar $Z$ via $Z_{, \alpha}=Z_{\alpha}$, for technical details and a more general treatment see Ref. [35]. It turns out that $Z=\zeta$ in canonical Weyl coordinates if the constant of integration is suitably chosen.
With this notation at hand, we can express the Weyl multipole moments by

$$
\begin{aligned}
U^{(r)} & =\int_{\mathcal{S}_{\mathcal{H}}} \eta_{a}^{(r)} \hat{n}^{a} d \mathcal{S}_{\mathcal{H}}+\int_{\mathcal{S}_{\mathrm{ext}}} \eta_{a}^{(r)} \hat{n}^{a} d \mathcal{S}_{\mathrm{ext}}, \\
\eta_{a}^{(r)} & =\frac{1}{8 \pi} \frac{\mathrm{e}^{U}}{W}\left(N_{-}^{(r)} U_{, a}-N_{+, W}^{(r)} Z_{, a} U+N_{+, Z}^{(r)} W_{, a} U\right),
\end{aligned}
$$

where $\hat{n}^{a}$ denotes the outward pointing unit normal to the surfaces $\mathcal{S}_{\mathcal{H}}$ and $\mathcal{S}_{\text {ext }}$ and the functions $N_{ \pm}^{(r)}$ depend on $(x, y)=(W, Z)$, see [35]. $d \mathcal{S}_{\mathcal{H}}$ and $d \mathcal{S}_{\text {ext }}$ are the proper area elements of $\mathcal{S}_{\mathcal{H}}$ and $\mathcal{S}_{\text {ext }}$, respectively. In vacuum, we can always choose canonical Weyl coordinates such that $W=\rho$ and $Z=\zeta$.

The induced multipole moments of distorted black holes.-With Eq. (10), we can identify the contribution of the different sources to the asymptotic Weyl multipole moments covariantly. The first term in Eq. (10), which we denote $U_{\mathcal{H}}^{(r)}$, gives the contribution of the distorted black hole and the second term, $U_{\mathrm{ext}}^{(r)}$, the contribution of the external sources. The induced multipole moment of a distorted black hole is now simply defined as $U_{\text {ind }}^{(r)}=$ $U_{\mathcal{H}}^{(r)}-U_{S}^{(r)}$, where the $U_{S}^{(r)}$ are the Weyl multipole moments of an undistorted Schwarzschild black hole. They coincide with the Newtonian multipole moments of a line mass of uniform density, see Ref. [32]. We parameterize $\mathcal{S}_{\mathcal{H}}$ for constant angles $\varphi$ from the "north pole" to the "south pole" $\left(s \in\left[s_{N}, s_{S}\right] \mapsto(\rho(s), \zeta(s), \varphi=\right.$ const $\left.)\right)$, cf. Ref. [35]. Then we obtain with Eq. (3) the $U_{\mathcal{H}}^{(r)}$ :

$$
\begin{aligned}
U_{\mathcal{H}}^{(r)}= & \frac{1}{4} \int_{s_{N}}^{s_{S}}\left[N_{-}^{(r)}\left(U_{S}+U_{D}\right)_{, n}\right. \\
& \left.-\left(N_{+, W}^{(r)} Z_{, n}-N_{+, Z}^{(r)} W_{, n}\right)\left(U_{S}+U_{D}\right)\right] d s,
\end{aligned}
$$

where we denote by $f_{, n}$ the normal derivative $-f_{, \rho}(\mathrm{d} / \mathrm{d} s)$ $\zeta(s)+f_{, \zeta}(\mathrm{d} / \mathrm{d} s) \rho(s)$. The multipole moments of a Schwarzschild black hole can be inferred from Eq. (11) by setting $U_{D}=0$. For the induced multipole moments, we have in turn only to subtract this Schwarzschild contribution from Eq. (11) and get

$U_{\text {ind }}^{(r)}=\frac{1}{4} \int_{s_{N}}^{s_{S}}\left[N_{-}^{(r)} U_{D, n}-N_{+, W}^{(r)} Z_{, n} U_{D}+N_{+, Z}^{(r)} W_{, n} U_{D}\right] d s$.

Applying the divergence theorem and Eq. (5), we can rewrite $U_{\text {ind }}^{(r)}$ :

$$
\begin{aligned}
U_{\text {ind }}^{(r)}= & \frac{1}{8 \pi} \int_{V_{\mathcal{H}}} \frac{1}{\rho}\left[U_{D, \rho}\left(N_{-, \rho}^{(r)}+N_{+, \zeta}^{(r)}-\frac{N_{-}^{(r)}}{\rho}\right)\right. \\
& \left.+U_{D, \zeta}\left(N_{-, \zeta}^{(r)}-N_{+, \rho}^{(r)}\right)\right] d V_{\mathcal{H}},
\end{aligned}
$$


which vanishes by virtue of Eq. (8). $V_{\mathcal{H}}$ is the coordinate volume enclosed by $\mathcal{S}_{\mathcal{H}}$ and $\mathcal{H}$ in canonical Weyl coordinates. Thus, the induced multipole moments vanish and the contribution of the distorted black hole to the asymptotic Weyl multipole moments is the same as that of a Schwarzschild black hole. With the results in Refs. [35,36], this can readily be translated to Geroch's multipole moments. This generalizes the no-hair theorem to black holes that are distorted by external matter. Note that this matter sources the gravitational field, too. Hence, the total asymptotic multipole moments differ in general from those of a Schwarzschild spacetime. This holds in particular for systems of two black holes. In the above derivation, the origin with respect to which the multipole moments are measured is chosen in the center of one of the black holes. Thus, the other black hole regarded as external matter contributes, for instance, a nonvanishing quadrupole moment to the total one, cf. the comment after Eq. (6).

The vanishing of the induced multipole moments implies that the second Love numbers $k_{r}$ vanish, too, because they are proportional to $U_{\text {ind }}^{(r)}$. This is corroborated by the results in Refs. [15,19-21]. But here we did not use any approximation or linearization. The result holds in full general relativity. Thus, one can assume $k_{r}=0$ for black holes, which rotate sufficiently slowly, in binary systems when calculating the emitted gravitational radiation during the adiabatic regime. Note that $k_{r}=0$ is specific to black holes. It does not hold for neutron stars, cf. Ref. [19]. Nonetheless, the source integrals of the Weyl multipole moments are still tailored to calculate their $k_{r}$, since the contributions from the individual sources to the Weyl multipole moments are separated covariantly and a definition of an induced multipole moment becomes possible in full general relativity. Moreover, it simplifies the evaluation of the source integrals in Ref. [35] in the presence of black holes considerably, since we have only to calculate the mass of the individual black holes to know all $U_{\mathcal{H}}^{(r)}$.

If the black holes rotate sufficiently slowly, there are several implications for astrophysics: On the one hand, measuring the mass of the black hole determines its contribution to the multipole moments completely. In binary systems containing a black hole or for a black hole with an accretion disc, the mass of the black hole can be inferred from the mass of the entire system measured by the motion of distant stars and the mass of the companion star or disc. After that all multipole moments of the black hole are fixed. Thus, every measurement of the multipole moments of the entire system, say, the quadrupole moment, determines the quadrupole moment of the companion or the disc. On the other hand, if the quadrupole moment of both, the entire system and the companion star or disc can be measured, then general relativity can be tested.

The multipole moments of the horizon.-Whereas the distorted black hole has the same asymptotic multipole moments as a Schwarzschild black hole, the horizon geometry clearly changes. This can be easily seen by evaluating the covariantly defined multipoles $M_{n}$ of isolated horizons following Refs. [11,12]. The scheme outlined therein was independently carried out in Ref. [13] using Schwarzschild-like coordinates. In that paper, it was found that the multipole moments of the distorted horizon are different from those of the Schwarzschild black hole. In fact, these deviations were used to define a relativistic analogue of the first Love numbers for black holes, which do not vanish in contrast to the second Love numbers.

The change in the geometry of the horizon is, however, not reflected in the asymptotic multipole moments. This, at first glance, counterintuitive behavior can be understood with a trivial Newtonian example. Consider a point mass and its multipoles. All multipole moments but the mass vanish and the equipotential surfaces are spheres. If an additional gravitational field generated by, say, a second point mass separated from the first is introduced, the multipole moments of the original point mass, which can be evaluated with Newtonian source integrals, are unchanged. In fact, the point particle has no inner structure and, thus, cannot be distorted by an external gravitational field and the source stays the same. Nonetheless, the equipotential surfaces are no longer spheres analogously to the distorted horizon. The situation changes of course, if an internal structure is assumed, like one would have to do for the description of neutron stars. Then the external gravitational field can indeed deform the matter distribution and the sources, which can be measured in the asymptotic multipole moments.

N.G. gratefully acknowledges support from the DFG within the Research Training Group 1620 "Models of Gravity". Partial support comes also from "NewCompStar", COST Action MP1304. The author thanks A. Ashtekar and J. Steinhoff for helpful discussions.

* Corresponding author. norman.guerlebeck@zarm.uni-bremen.de

[1] C. Done, C. Jin, M. Middleton, and M. Ward, Mon. Not. R. Astron. Soc. 434, 1955 (2013).

[2] C. S. Reynolds, Classical Quantum Gravity 30, 244004 (2013).

[3] G. Risaliti et al., Nature 494, 449 (2013).

[4] F. Eisenhauer et al., in Science with the VLT in the ELT Era, GRAVITY: Microarcsecond Astrometry and Deep Interferometric Imaging with the VLT, edited by A. Moorwood (Springer Netherlands, Dordrecht, 2009), p. 361.

[5] Z. Li and C. Bambi, J. Cosmol. Astropart. Phys. 01 (2014) 041.

[6] A. Grenzebach, V. Perlick, and C. Lämmerzahl, Phys. Rev. D 89, 124004 (2014).

[7] S. S. Doeleman et al., Nature 455, 78 (2008).

[8] A. E. Broderick, T. Johannsen, A. Loeb, and D. Psaltis, Astrophys. J. 784, 7 (2014).

[9] T. Johannsen and D. Psaltis, Astrophys. J. 773, 57 (2013). 
[10] M. Wade, J. D. E. Creighton, E. Ochsner, and A. B. Nielsen, Phys. Rev. D 88, 083002 (2013).

[11] A. Ashtekar, J. Engle, T. Pawlowski, and C. v. d. Broeck, Classical Quantum Gravity 21, 2549 (2004).

[12] A. Ashtekar and B. Krishnan, Living Rev. Relativity 7, 10 (2004).

[13] T. Damour and O. M. Lecian, Phys. Rev. D 80, 044017 (2009).

[14] E. E. Flanagan and T. Hinderer, Phys. Rev. D 77, 021502(R) (2008).

[15] T. Damour and A. Nagar, Phys. Rev. D 80, 084035 (2009).

[16] R. Geroch and J. B. Hartle, J. Math. Phys. (N.Y.) 23, 680 (1982).

[17] T. Hinderer, Astrophys. J. 677, 1216 (2008); 697, 964(E) (2009).

[18] A. Love, Some Problems of Geodynamics (Cornell University Library, Ithaca, 1911).

[19] T. Binnington and E. Poisson, Phys. Rev. D 80, 084018 (2009).

[20] B. Kol and M. Smolkin, J. High Energy Phys. 2 (2012) 10.

[21] S. Chakrabarti, T. Delsate, and J. Steinhoff, arXiv: 1304.2228.

[22] C. P. L. Berry, I. Mandel, H. Middleton, L. P. Singer, A. L. Urban, A. Vecchio, S. Vitale, K. Cannon, B. Farr, W. M. Farr, P. B. Graff, C. Hanna, C.-J. Haster, S. Mohapatra, C. Pankow, L. R. Price, T. Sidery, and J. Veitch, arXiv:1411.6934.

[23] L. Baiotti, T. Damour, B. Giacomazzo, A. Nagar, and L. Rezzolla, Phys. Rev. Lett. 105, 261101 (2010).
[24] J. S. Read, L. Baiotti, J. D. E. Creighton, J. L. Friedman, B. Giacomazzo, K. Kyutoku, C. Markakis, L. Rezzolla, M. Shibata, and K. Taniguchi, Phys. Rev. D 88, 044042 (2013).

[25] K. Yagi and N. Yunes, Science 341, 365 (2013).

[26] K. Yagi and N. Yunes, Phys. Rev. D 88, 023009 (2013).

[27] A. Maselli, V. Cardoso, V. Ferrari, L. Gualtieri, and P. Pani, Phys. Rev. D 88, 023007 (2013).

[28] D. D. Doneva, S. S. Yazadjiev, N. Stergioulas, and K. D. Kokkotas, Astrophys. J. Lett. 781, L6 (2014).

[29] B. Haskell, R. Ciolfi, F. Pannarale, and L. Rezzolla, Mon. Not. R. Astron. Soc. 438, L71 (2014).

[30] S. Chakrabarti, T. Delsate, N. Gürlebeck, and J. Steinhoff, Phys. Rev. Lett. 112, 201102 (2014).

[31] G. Pappas and T. A. Apostolatos, Phys. Rev. Lett. 112, 121101 (2014).

[32] H. Stephani, D. Kramer, M. MacCallum, C. Hoenselaers, and E. Herlt, Exact Solutions of Einstein's Field Equations (Cambridge University Press, Cambridge, England, 2003).

[33] J. M. Bardeen, in Black Holes (Les Astres Occlus), Rapidly Rotating Stars, Disks, and Black Holes, edited by C. Dewitt and B.S. Dewitt (Gordon and Breach Science Publishers, New York, 1973).

[34] B. Carter, in Black Holes (Les Astres Occlus), Black Hole Equilibrium States, edited by C. Dewitt and B. S. Dewitt, (Gordon and Breach Science Publishers, New York, 1973), pp. 57-214.

[35] N. Gürlebeck, Phys. Rev. D 90, 024041 (2014).

[36] G. Fodor, C. Hoenselaers, and Z. Perjés, J. Math. Phys. (N.Y.) 30, 2252 (1989). 